A N N A L E S Annales de Bretagne et des Pays de l'Ouest

Anjou. Maine. Poitou-Charente. Touraine

$114-4$ | 2007

Varia

\title{
1720-1721 : la peste ravage Toulon
}

Conséquences démographiques et économiques

Michel Vergé-Franceschi

\section{(2) OpenEdition Journals}

Édition électronique

URL : http://journals.openedition.org/abpo/461

DOI : $10.4000 / a b p o .461$

ISBN : 978-2-7535-1508-6

ISSN : 2108-6443

Éditeur

Presses universitaires de Rennes

Édition imprimée

Date de publication : 30 décembre 2007

Pagination : $57-71$

ISBN : 978-2-7535-0598-8

ISSN : 0399-0826

Référence électronique

Michel Vergé-Franceschi, «1720-1721 : la peste ravage Toulon », Annales de Bretagne et des Pays de l'Ouest [En ligne], 114-4 | 2007, mis en ligne le 30 décembre 2009, consulté le 19 avril 2019. URL : http://journals.openedition.org/abpo/461 ; DOI : 10.4000/abpo.461 


\title{
1720-1721 : La peste ravage Toulon Conséquences démographiques et économiques
}

\author{
Michel VerGÉ-FRANCESCHI \\ Professeur d'Histoire moderne, \\ CEHVI - Université de Tours
}

Toulon connaît les épidémies depuis toujours : 1348, 1581, 1587, 1664, d'où la création de Bureaux de Santé à Marseille et Toulon, ce dernier dès 1576. Celui-ci instaura à Lagoubran, à l'ouest de Toulon, un lazaret qui devait permettre de faire accoster les bateaux dans un abri jusqu'à la fin de leur mise " en quarantaine ", de décharger les cargaisons en assurant leur désinfection soit par la " sereine " (aération), soit par le " parfum " (brûlage d'aromates), d'assurer la logistique des équipages mis en quarantaine (vivres, soins). Dès 1622, les consuls songèrent à établir un nouveau lazaret " à l'isle de Sepet " à Saint-Mandrier, presqu'île qui fait face à la rade de Toulon. Ils achetèrent un terrain à flanc de coteau, au milieu de la garrigue, le long de la mer et le lazaret fut construit à partir de 1657 « à l'extrémité de la grande rade, entre l'hôpital de Saint-Mandrier et les Sablettes ". Celui-ci fut correctement aménagé : quai de débarquement de la Santé, cour d'entrée de la Santé " servant à intercepter la communication du quai de la Santé ", logement du capitaine de la Santé, jardin potager à son usage, réservoir à poissons, vestibule d'entrée, chapelle, baraques, entrepôts et hangars, " chauffoirs où l'on parfume les équipages en quarantaine pour leur donner l'entrée ", "grand parloir pour les équipages en quarantaine ", " loge pour les bestiaux ", " grand puits ", " enclos ", " cimetière des catholiques, cimetière des huguenots ", outre une trentaine d'arbres peut-être à valeur médicinale. Tout est prévu, officiellement pour " parquer " les équipages à risques et les inhumer. Mais, au XVIII ${ }^{\mathrm{e}}$ siècle encore, un anonyme écrit : "le lazaret est dénué de tout secours de bâtiments propres à rétablir la santé des équipages des vaisseaux du Roi et autres bâtiments, comme sont un hôpital ou des infirmeries pour y retirer les convalescents ". Le lazaret est en réalité plus un mouroir qu'un établissement de soin. La peste de 17201721 le prouva. 


\section{Les mesures de précaution}

À Toulon, les consuls étant automatiquement "intendants de la santé " après leur sortie de charge, il leur revient d'établir les cordons sanitaires et de faire vérifier, aux portes de la ville ou sur les lieux de leur débarquement, les «billets" de santé des voyageurs. La Consigne, ou Bureau de la Santé, sise dans un bâtiment du port, avait l'habitude de recevoir les déclarations des capitaines ; tout capitaine arrivant au port se devait en effet de présenter une " patente " qui pouvait être " nette " (aucun danger), " susceptible " (danger possible), ou "brute " (si une maladie s'était déclarée à bord au cours de la traversée). Mais rien ne put endiguer le fléau.

À Toulon, en 1720, le premier consul est Jean Geoffroy (1693-1762), issu d'un tabellion d'Antrechaux connu en 1453. Les Geoffroy sont arrivés à Toulon avec le bisaïeul du consul, premier Geoffroy d'Antrechaux inhumé jadis dans le cloître des minimes. Le grand-père du consul, Jean, est mort à Toulon en 1712 dans une maison édifiée en 1690 face à la colonnade ouest de la Halle aux Poissons élevée par Pierre Puget. Le père du consul, Jacques, négociant en soierie, a acheté en 1704 la charge anoblissante de " secrétaire du Roi en la chancellerie du parlement de Provence ". Il est mort en 1705 dans sa maison rue aux Arbres (actuel n ${ }^{\circ} 91$ cours La Fayette), achetée en $1696^{1}$. Suite à ses mauvaises affaires, ses meubles furent vendus aux enchères place Saint-Michel, devant la maison (actuelle place Hubac). En 1720, Jean d'Antrechaux est un consul de vingt-sept ans, jeune marié, dynamique et déterminé, qui vit entouré de ses tantes, Tante "Nanon ${ }^{2}$ " qui l'éleva, $\mathrm{M}^{\mathrm{me}}$ Tournier ${ }^{3}$, épouse du conseiller de ville Hyacinthe Tournier, $\mathrm{M}^{\mathrm{me}}$ Pierre de Selves ${ }^{4}$. Le consul a quatre frères, un oncle et un grand-oncle du nom; il a aussi un grand-oncle, Joseph d'Antrechaux, chevalier de Malte, mais la descendance illégitime de celui-ci, issue d'une belle grecque (Callizza), est restée dans un couvent aux Sporades ${ }^{5}$.

En 1720, la peste sévit à Marseille dès juillet. Le 11 de ce mois, les intendants de la santé de Toulon l'apprennent. Aussitôt, avec compassion et inquiétude, ils adressent leurs condoléances aux intendants de la santé de Marseille. Mais surtout, on devient extrêmement vigilant : quelques barques arrivant de Marseille à Toulon sont immédiatement refoulées, par crainte de " la contagion ". Lorsqu'elles deviennent trop nombreuses, la Marine les chasse grâce aux vaisseaux de guerre de Sa Majesté qui peuplent la rade, dite « darse Vauban » depuis les travaux qu'il y réalisa dans les années 1680. Le 31 juillet, Jean d'Antrechaux sort de sa maison rue aux Arbres et préside à l'hôtel de ville un conseil extraordinaire, en présence des autorités militaires et des notables du port. Un "bureau de santé " est immédiatement formé; deux intendants de santé supplémentaires sont nommés, s'ajoutant

1. Ganteaume, notaire.

2. Anne d'Antrechaux, morte à 73 ans le 10 oct. 1747 .

3. Catherine d'Antrechaux, morte le 22 oct. 1747.

4. Madeleine d'Antrechaux, morte le 13 oct. 1747.

5. Catherine d'Antrechaux, sœur Rose en religion. 
aux six déjà existant; un cordon sanitaire est mis en place; la quarantaine est renforcée. Mais le lazaret compte déjà ses premiers pestiférés alors que Bandol (entre Marseille et Toulon) commence aussi à être infecté. Toulon s'inquiète, s'active et prépare ses hôpitaux à Saint-Mandrier, puis au quartier Saint-Roch, le saint protecteur de la peste, régulièrement fêté le 16 août, partout en Méditerranée (en Corse notamment). Partout les femmes viennent s'agenouiller devant sa statue sur laquelle, de sa main droite, il désigne généralement un bubon sur sa jambe. Les femmes pleurent et prient. Les hommes tremblent. Mais deux mois s'écoulent (11 juillet-10 septembre) sans trop de désastres, si ce n'est les habituels malheurs toulonnais dus aux fortes chaleurs estivales : odeurs insupportables de la vieille darse (ou darse Henri IV), réservée aux navires marchands, odeurs fétides de toutes les rues du port, qui servent en même temps d'égout à ciel ouvert, égout seulement nettoyé par les pluies d'automne, les rues en pente, toutes orientées du Faron vers la mer, conduisant les eaux de ruissellement et les déjections dans le port. Mais l'été, les ordures et les excréments des chevaux et des hommes s'entassent au fond des impasses, véritables " sueilles » où le crottin fermente, mélangé aux ordures ménagères, sous des températures souvent supérieures à $30^{\circ} \mathrm{C}$. L'eau est souvent corrompue. Les rivières voisines sont asséchées, les fontaines malsaines.

Le 10 septembre, les choses commencent à changer : ce jour-là, Gérin, beau-père du consul d'Antrechaux, embarque à La Ciotat avec l'avocat Gaudemar, toulonnais (parent des Colbert), procureur du Roi, le commis au greffe Viennot et l'huissier Drogue. Tous trois gagnent l'île de Jarros avec quelques commis aux écritures et autres " écrivains de Marine ", et, dix jours durant, ils inventorient avec minutie les ballots du Grand Saint-Antoine avant de les faire brûler, la cargaison de ce navire marchand étant fortement incriminée. On reproche sur toute la côte, de Marseille à Toulon, au capitaine Chataud, d'avoir débarqué en fraude des étoffes, alors que la peste sévissait à son bord. Reproche fréquent et généralement fondé : les capitaines marchands, répugnant à perdre leur cargaison, préfèrent généralement taire l'existence du fléau à leur bord. On va parfois jusqu'à jeter à la mer les corps des malades morts au cours de la traversée afin d'obtenir, une fois arrivé au port, une patente " saine " alors que l'on a eu des morts à bord! Chataud, interrogé, obtient toutefois un non-lieu... mais sept des compagnons de Gérin meurent - comme par hasard -, les jours suivants. Gérin assume seul la charge financière de l'enquête, huit cents livres, et il doit bientôt assurer une pension annuelle de cent cinquante livres à la veuve Viennot qui l'accuse d'avoir entraîné son époux vers une mort prématurée.

\section{Le développement de l'épidémie à Toulon}

\section{Les débuts et les premières réactions}

Au début d'octobre, les premiers cas de peste apparaissent à Toulon et mi-octobre, les premiers morts. Énergique, le jeune consul fait visiter 
les maisons; il fait approvisionner la ville en denrées, depuis Lorgues ou l'Auvergne. En décembre, le fléau se renforce. Des mesures draconiennes sont prises : les fêtes de Noël sont supprimées. Pauvres et ouvriers s'en plaignent, en raison de l'absence de " charités publiques".

Le 2 janvier 1721, les intendants de la santé tentent néanmoins de se rassurer : "La santé est très bonne [...] Nous avons eu deux attaques du mal, mais nous avons eu le bonheur de le couper. "Quinze jours passent. Mais le 17, meurt la fille du colporteur Gras, considéré comme l'introducteur de la peste pour avoir apporté des étoffes, débarquées en fraude du Grand Saint-Antoine. Aussitôt, les notables paniquent et fuient " à la campagne " en leurs châteaux voisins du port et autres bastides rurales. L'air y est reconnu comme moins "vicié " qu'à Toulon. De vastes espaces de verdures s'offrent à eux : garrigues et maquis paraissent plus sains que les rues étroites et tortueuses de Toulon. Le " bon air " est celui d'Ollioules, du Beausset, de La Cadière, des seigneuries voisines du port, à une ou deux lieues de celui-ci. Ainsi, les Thomas à La Valette, officiers des vaisseaux du Roi et seigneurs de La Valette à une lieue de Toulon, gagnent-ils leur château de La Valette, à l'est du port, voisin du château de La Garde (où mourra le marquis d'Argens), propriété de leurs cousins Thomas de La Garde, et de la terre de Châteauneuf, terre de leurs cousins Thomas de Châteauneuf. Joseph de Thomas "marquis " de La Valette (1672-1744), officier des vaisseaux du Roi, se fait affecter "à La Valette", dans son propre fief, " par ordre de M. Duquesne-Mosnier " aux ordres duquel il avait navigué en 1714, 1717-1718, 1718-1719. Chargé d'" y contenir les soldats de la marine " qui s'y trouvaient, il en profita pour éloigner de Toulon son fils unique, qu'il finira par enterrer, mais en 1736 seulement. Le « marquis " n'est rentré à Toulon qu'au quatrième trimestre 1721, la guérison proclamée.

Fils et petit-fils de consuls de Toulon, l'officier de marine Martini d'Orves (futur lieutenant général des armées navales, co-seigneur d'Orves à l'ouest de Toulon, et oncle des d'Estienne d'Orves), demeure «à la ville ». Le port a en effet besoin de ses notables : la ville manque de pain, de viande, d'argent, de secours matériels et spirituels. Le 28 janvier, la " quarantaine générale " est déclarée : il est décidé que les Toulonnais resteront désormais cloîtrés chez eux et qu'ils seront nourris à domicile par six cents " pourvoyeurs " (souvent des galériens) qui se rendront dans les huit quartiers et faubourgs. Les « huit mille indigents " du port (?), chiffre énorme qui représenterait le tiers de la population toulonnaise (?), devront être entretenus " gratis". Les consuls reçoivent les pleins pouvoirs et s'établissent à l'hôtel de ville pour y demeurer aux commandes, nuit et jour. Le maintien de l'ordre est remis aux troupes réglées. Tous les médecins du port sont réquisitionnés (environ trois ou quatre praticiens), de même tous les chirurgiens.

En février, les morts remplissent un tombereau quotidien de cinquante places. La sinistre charrette passe dans les rues étroites chaque jour. On l'écoute sur le pavé glissant. On la craint. On la redoute. On y jette souvent les cadavres depuis les fenêtres des étages, enveloppés d'un simple drap 
ou « linceul " blanc. Les galériens sont souvent chargés de cette sinistre besogne et certains d'entre eux en profitent pour dérober quelqu'objet chez le mort. Moindre mal. Il leur arrive parfois de jeter dans le tombereau un malade, en fin de vie certes mais pas encore tout à fait mort, et le malheureux agonisant est condamné, pour les derniers moments de son existence ici-bas, à voisiner avec des corps déjà en putréfaction. En février aussi, la peste éclate à La Valette. En mars, à Toulon, deux tombereaux deviennent nécessaires pour faire évacuer les cadavres, cent morts par jour, par les " corbeaux ". D'Antrechaux, au cimetière, s'active et fait creuser des " saloirs " où on empile les corps pêle-mêle. Les intendants de santé débordés par le fléau sont violemment pris à partie par la population en fureur qui cherche des coupables faute de remèdes. Le 12, ils se défendent d'avoir eu aucune complaisance au sujet des quarantaines. Mais le non-lieu qui a « blanchi » Chautard continue à indigner les esprits, même si les corps sont de plus en plus affaiblis. La Marine organise son périmètre de sécurité et prend une décision originale puisqu'elle décide d'enfermer les " jeunes ", c'est-à-dire les gardes de la marine (futurs enseignes des vaisseaux du Roy), au " Jardin du Roi ", sous l'autorité du chevalier de Piosin, chevalier de Malte toulonnais (futur bailli de son ordre), officier des vaisseaux du Roi (futur chef d'escadre) et cousin germain de Martini d'Orves. Tous ces gardes de la marine, espoirs de la Marine de demain, survivront, à l'exception d'un Moscovite, envoyé à Toulon par Pierre le Grand, imprudemment sorti du " Jardin du Roi ». Il est vrai que les Russes avaient, face à la peste, un remède aussi réel qu'étonnant : ayant remarqué que la peste était véhiculée par les puces des rats et ayant remarqué que les puces ne piquaient point les chevaux, les nobles russes avaient pris l'habitude de rester, nuit et jour, à cheval pendant la durée des épidémies, nourris par leurs domestiques restés à terre, grâce à de longues perches!

\section{L'épidémie à son apogée}

En avril, à Toulon, quatre tombereaux quotidiens s'avèrent nécessaires : deux cents morts par jour; deux cent soixante-dix morts sont même à déplorer pour le seul 30 avril. Le 16 avril, le commandant de la Marine Abraham Duquesne-Mosnier (neveu du Grand Duquesne) parlant de Louis Beaussier, lieutenant de port depuis 1703, loue "sa capacité, son zèle pour le service et son arrangement admirable ${ }^{6}$ ". Début mai, huit tombereaux quotidiens deviennent à présent indispensables : quatre cents morts quotidiens. L'épidémie culmine. Des rues entières sont dépeuplées. Vides, les immeubles résonnent du seul pas des pillards, mi-voleurs, mi-charognards. Des médecins viennent de Marseille, Montpellier, Paris, car tous leurs collègues toulonnais, sauf un, sont morts. Les forçats sont envoyés depuis Marseille pour ensevelir les cadavres qui, faute d'hommes valides, pourrissent en plein air. L'hôpital de la Charité est un véritable mouroir où

6. Arch. du port de Toulon, Duquesne-Mosnier à la Cour, 16 avril 1721. 
les gémissements des agonisants l'emportent sur les pleurs de ceux qui auraient dû verser des larmes sur eux, mais qui sont déjà morts. En vain, on cherche des remèdes. On pend à l'occasion quelques galériens et quelques filles de joie pour tenter d'exorciser le mal. Ici, un capucin multiplie les prêches. Là, l'évêque de Toulon, M ${ }^{\text {gr }}$ de Piosin-Montauban, voue Toulon au Sacré-Cœur. Vœu exaucé. Le 10 mai, la « serrade " est enfin levée.

\section{Les premières conséquences}

Mais Toulon se réveille sous un vrai cauchemar. Le 20, par délibération du conseil de ville, Louis de Martini d'Orves est nommé " commissaire général pour la santé [...] pour servir conjointement avec Messieurs les consuls, avec permission de M. Duquesne-Mosnier ". Les déserteurs, contraints hier d'enterrer les cadavres, meurent aujourd'hui presque tous, à l'issue de leur période d'incubation. La mort des boulangers prive Toulon de pain. Toutes les institutions municipales sont bouleversées; en juin, on doit surseoir au renouvellement du corps de ville : les consuls Henri Marin et Gabriel Gavoty sont morts; ont succombé les conseillers de ville Hyacinthe Tournier, Antoine Serre, Joseph Richard, tous les capitaines de quartiers, trois des huit intendants de la santé, huit des quatorze sage-femmes. Sont morts les soldats de l'Île-Royale ou de Brie, malheureusement en garnison à Toulon. D'Antrechaux a perdu ses trois frères et tous ses domestiques : Nicolas-Antoine d'Antrechaux, frère minime, est mort à vingtdeux ans le 25 octobre 1720 à Marseille; Antoine d'Antrechaux, lieutenant au régiment de Ponthieu et Joseph d'Antrechaux, jeune officier au même régiment, sont morts à Toulon en juillet 1721, alors que le consul leur avait défendu de venir l'y rejoindre; Paule d'Antrechaux, sa sœur, religieuse ursuline, est morte elle aussi, dans son couvent, avec ses cousines; ses oncles paternels ont succombé : Hyacinthe Tournier et Antoine d'Antrechaux sont morts à l'hôtel de ville, le premier, conseiller de ville, le second membre de l'Assemblée générale de la communauté; son grand-oncle, le père jésuite Joseph d'Antrechaux, professeur de logique, de métaphysique, régent de rhétorique au collège Bourbon d'Aix, est mort à cinquante ans en venant secourir les Toulonnais qui l'avaient vu naître.

\section{La fin de l'épidémie}

Fin juillet, le fléau décroît. La quarantaine est enfin supprimée. Les pouvoirs du seul consul survivant, d'Antrechaux, prorogés le 16 juin, le sont à nouveau le 24 juillet alors que la sècheresse sévit. D'Antrechaux prend un arrêté : «Un fontanier municipal sera invité à faire couler le plus d'eau possible dans les rues et quarante mendiants seront engagés comme balayeurs, chacun étant payé deux sols par jour ". En août, les gardes de la marine cessent d'être consignés au " Jardin du Roi ». Le 7 août, Claude de Beaucaire, officier des vaisseaux du Roi originaire de Moulins, et qui commandait jusqu'alors " le militaire dans le parc et dans l'arsenal ", quitte 
l'arsenal et se dévoue " à la ville ». Le 15, les survivants se comptent. Le 20, les intendants de santé dressent un premier bilan : « Du côté de l'ouest, il y a La Seyne et Ollioules qui ont été fort ravagés mais aujourd'hui le mal y a presque cessé; il s'est aussi répandu aux hameaux de Six-Fours et à SaintNazaire (Sanary), depuis vingt ou vingt-cinq jours. Le Revest a été aussi mal traité que La Seyne et Ollioules. Du côté du Levant, jusqu'à présent, il n'y a que Neoulles, Gareou, La Foux du Puget, Saint-Estayié, Roquabaron, Fourquanquerel, La Valette où le mal a été si violent qu'il n'y a eu qu'une ou deux maisons exemptes du mal qui y a fini depuis la Saint-Jean (24 juin), et La Garde qui a été aussi maltraitée que La Valette ». Le dernier pestiféré meurt à Toulon le 7 septembre.

Le 25 octobre, d'Antrechaux et les conseillers de ville Portallis, André Tournier, Creissel, Dorves écrivent à Marseille à M. de Langeron, officier des galères : "Il est de notoriété publique que nous n'avons eu ni morts ni malades depuis le 18 du mois d'août ". (Faux, il y a eu un mort le 7 septembre); « On a fait en cette ville des réjouissances pour le rétablissement de la santé du Roi "; " On a donné l'entrée dans Toulon à quinze cents hommes qui étaient enfermés dans l'Arsenal "; " Une partie des soldats et des ouvriers de la Marine ont rejoint leurs femmes dans un état encore de convalescence et les autres se sont remariés "; " Puisque vous nous déclarez que votre terroir est aussi sain que la ville, nous vous déclarons que le nôtre est bien sain aussi "; « La dernière attaque est du 7 de septembre en la personne d'un Bonnegrasse qui mourut de la peste au quartier des Routes, il y a aujourd'hui quarante-huit jours "; « Notre lazaret [...] est occupé par des convalescents qui sont sortis de l'hôpital de la Charité et qui y finissent leur quarantaine "; « Notre hôpital de la Charité est fermé. Il nous reste, il est vrai, dix-neuf malades que nous avons fait passer à SaintRoch pour les éloigner de la ville, mais ce sont de vieux malades qui sont devenus incurables et qui ne peuvent même plus communiquer leurs maux qui sont dégénérés en fistules et en phtisie. [...] Le Seigneur délivrera les nôtres quand il lui plaira ".

Le 30 octobre, un Te Deum réunit les survivants. Le 25, Martini d'Orves a reçu en récompense de ses efforts six cents livres de pension sur le Trésor royal, outre le grade de capitaine de compagnie franche. Le 7 novembre, une déclaration officielle sanctionne la guérison : c'est " l'Acte déclaratif de l'état de la Santé dans la ville de Toulon, dans son territoire, et dans tous les lieux de la viguerie qui ont été affligés de la contagion ». Peu après, d'Antrechaux écrit : " un acte de religion, d'amour et de reconnaissance ne nous permit pas de différer le service funèbre que nous devions à la mémoire des deux consuls morts au service de la Patrie. M. l'évêque voulut bien y officier pontificalement. On avait élevé un catafalque lugubrement orné. Dans chacun des angles, on lisait sur des écussons aux armes de la Ville : Huc nos Patriae pietas. Nos citoyens et tous les corps militaires honorèrent ce service de leur présence. J'étais alors seul consul et j'assistai à cette pompe funèbre sans marque distinctive, sans faste et sans cortège ». Le 23 décem- 
bre, Louis de Martini d'Orves reçoit la croix de Saint-Louis en même temps que le lieutenant de port Louis Beaussier, promu dès 1722 capitaine de vaisseau et de port. À La Valette, le Père Bastide, de l'ordre des minimes, rescapé de la peste qui l'a pourtant atteint, succède au curé Poumet mort de la " contagion ». Sur 1598 habitants, La Valette en conserve 1068, après la mort de 530 pestiférés emportés entre février et octobre 1721, soit un Valettois sur trois. Il faut attendre 1765 pour que La Valette compte 1612 habitants. Au Revest, sur 500 habitants, 300 sont morts, dont les deux premiers consuls, le troisième, Sauvaire, signe la fin de la contagion le 7 novembre 1721 mais décide de faire garder le poste du bois d'Orves pour prévenir toute arrivée de personne étrangère au Revest. Il faut attendre 1770 pour que Le Revest compte à nouveau 463 habitants.

Le 8 janvier 1722, d'Antrechaux sort de charge et le Roi le nomme viguier de Toulon pour deux ans et demi, selon la tradition. Il retrouve sa femme Claire de Gérin (Toulon 1701-Toulon 1727) et leur fille ${ }^{7}$ d'un an, réfugiées en leur bastide du Pont-du-Las " les Marronniers "; le consul et son épouse eurent ensuite plusieurs enfants ${ }^{8}$ mais Claire fut emportée par une fausse couche. D'Antrechaux reçut une série de félicitations : lettres du maréchal de Villars, gouverneur de Provence (21 juillet 1721), de M. Le Blanc, ministre de la guerre (24 juillet). D'Antrechaux profita quelque peu de la situation pour faire recevoir son frère cadet Pierre, vingt ans, lieutenant au régiment de Ponthieu. Le 3 mai 1723, le Roi accorda à l'ancien consul mille livres de pension sur le Trésor royal et le 7, il lui offrit le cordon de Saint-Michel. Son beau-père Gérin le reçut en même temps, pour avoir continué à remplir ses fonctions de magistrat pendant la peste : du 28 juin 1720 au 14 septembre 1721, Gérin a rendu 128 sentences qui occupent 1148 pages in-octavo de papier timbré, plus les insinuations des actes intéressant l'Amirauté. En effet, comme durant toute épidémie, les vols ont été légion à Toulon, nombre de " corbeaux " ne se contentant pas d'emporter les corps des pestiférés! Le consul et son épouse eurent alors plusieurs enfants ${ }^{9}$ mais Claire fut emportée par une fausse couche.

\section{Les conséquences}

À Toulon, les élites, qui ont été peu touchées par l'épidémie, se recomposent après la peste. Le 22 septembre 1722, Claude de Beaucaire (1660-1735), capitaine de vaisseau célibataire, épouse une jeune veuve : Agnès Clapier, fille de Jean " bourgeois d'Hyères " et de Marguerite Emeric, et veuve de François Monier avocat à la cour, laquelle maigrement dotée - quinze mille livres -, a déjà deux filles. En la cathédrale Sainte-Marie, Beaucaire, gentilhomme et officier d'épée, s'entoure pour épouser cette veuve d'avocat de quatre témoins : Henry de Modène, chef d'escadre, Charles de Vallette-

\footnotetext{
7. Claire-Madeleine.

8. Jean-Charles (1723), une fille (1724), Jean-Joseph (1726).

9. Jean-Charles (1723), une fille (1724), Jean-Joseph (1726).
} 
Laudun, futur chef d'escadre, Pierre Charronier, commissaire général et Jean-Baptiste de Vence, capitaine de vaisseau. En 1724, Louis de La Tour d'Auvergne, comte d'Évreux, est parrain de sa première fille; en 1727, l'intendant Mithon est parrain de la deuxième; en 1732, le ministre de la marine Maurepas est parrain par procuration de son fils aîné prénommé JeanFrédéric en hommage au ministre. La même année, Beaucaire, jeune père de soixante et douze ans, marie la fille aînée de son épouse, Anne Monier, fille d'avocat, petite-fille de bourgeois, au futur capitaine de vaisseau Jacques Gabriel de Burgues de Missiessy, noble et officier d'épée; et lorsque (1741) Beaucaire meurt à soixante et quinze ans, terrassé par deux attaques d'apoplexie juste après l'ondoiement de son sixième enfant, sa veuve marie son autre fille Monier à Joseph-Marie de Ruyter qui passe à Toulon pour être un descendant du grand amiral hollandais Michel de Ruyter, (ce qui est une pure légende sans aucun fondement généalogique).

La peste a changé la physionomie de Toulon. Élites bourgeoises et maritimes se respectent comme elles ne se sont jamais respectées. Le premier consul d'Antrechaus écrit dans ses Mémoires publiés ultérieurement :

"Je ne dois pas passer sous silence les services rendus par MM. les officiers de la Marine dont j'ai moi-même déposé les preuves dans les archives de la ville de Toulon. Ces messieurs qui ne connaissent l'inaction que lorsqu'il ne leur est point libre d'en sortir, se disputèrent entre eux à qui se rendrait plus utile à la ville pendant la quarantaine générale; ils se firent une gloire de la soutenir dans son désastre, de l'assister de leurs soins, et de nous aider de leurs sages conseils. On eût dit que l'État les rendait responsables du salut de cette ville."

Les officiers morts sont pourtant peu nombreux, une douzaine : le capitaine de vaisseau Grimaudet de Motheux et Joseph de Terras d'Orgon ${ }^{10}$; le lieutenant de vaisseau Pierre de Beaussier de Quiez neveu du vieux Félix de Beaussier (capitaine de vaisseau) qui traverse l'épidémie, les lieutenants de vaisseau de Chabert-Taillard, de Gineste, de Grandmaison, de Forbin-SainteCroix, de la Faudré, de Saint-Julien de Monderville, le Fann; les gardes du pavillon Thomas d'Orves, de Baillibaud, de Saint-Maux. Mais les officiers retirés " dans leurs campagnes " fournirent des secours matériels à la ville. En 1731, le trésorier de la communauté de Toulon rembourse aux héritiers de Pierre Beaussier de Quiez, deux mille livres prêtées par ce dernier pour soulager les misères liées à la " contagion ". De même, il rembourse à Joseph de Catelin de La Garde, officier de vaisseau, deux mille livres sur les onze mille que la communauté lui doit ${ }^{11}$.

10. Fils de Joseph de Terras d'Orgon, capitaine de brûlot (1702), de vaisseau (16951696), ou de frégate (1710), chevalier de Saint-Louis. Frère de Gabriel, officier des galères, marié à Toulon (1715). Son père avait épousé en premières noces la fille du chef d'escadre de Cuers-Cogolin, avant de se remarier à Marguerite de Martin (Arch. nat., C7 88 et Arch. com. de Toulon, acte de baptême du 5 nov. 1697).

11. Arch. com. de Toulon, BB 85, 17 déc. 1731 et 20 oct. 1733. 
La peste fut une vraie catastrophe, démographique et culturelle; quantité de "savoirs " ayant été emportés : savoirs techniques des ouvriers de l'arsenal, des calfats, charpentiers, ferronniers, peintres et artistes issus de l'école de Puget. En outre, les bois entrés dans l'arsenal en 1718, trentetrois mille pièces, coupés dans leur sève, ont pourri sur place, faute de bras. En 1723, il ne reste plus que onze cent cinquante pièces bonnes! Le constructeur François Chapelle cherche à acheter la coupe des arbres des Pontevès pour y remédier. Mais, " esprit brouillon, il a aigri les propriétaires par des hauteurs insupportables ${ }^{12}$ ". Toulon est au plus bas : le commerce ruiné, l'artisanat en déroute; la savonnerie s'enlise, la culture des câpres décline, l'activité de l'arsenal n'a rien à voir avec celle des années 1680 qui marquent l'apogée du port : il y avait alors quinze tanneries, vingt fabriques de savon, douze de chapeaux, dix manufactures de draps grossiers ou pinchinats, huit de cotonnerie ou toile à voile, des fabriques de chandelles, de cierges, de la distillerie. Cette ruine de Toulon est toutefois exagérée par les contemporains qui prétendent qu'au XVII e siècle Toulon vivait d'un commerce actif. Or tous les témoins, dont le président de Séguiran (d'Aixen-Provence), déploraient le peu de commerce fait par Toulon à l'époque de Richelieu (1635). Ce n'est pas le grand négoce que la peste a ruiné : il n'a jamais existé à Toulon. C'est plutôt le monde des boutiquiers. Par manque de bras, la vallée de Dardennes reprend mal son activité avec ses paroirs à draps, ses forges, ses moulins à huile et à farine.

La ruine de Toulon est autant morale qu'économique. Les survivants d'un peuple décimé jalousent les élites restées intactes ou habilement recomposées. Les " bastides rurales " ont offert le " bon air ", "l'altitude " d'Évenos, du Beausset ou de La Cadière, donc la survie à leurs occupants aisés alors que le menu peuple pauvre, vivant dans les rues étroites de Toulon, a fini par s'entasser, mort, dans les effroyables tombereaux. Malgré les solidarités financières, rancoeurs et jalousies sont nombreuses. D'Antrechaux est traqué par un de ses successeurs qui lui demande des comptes sur sa gestion estimée trop dispendieuse. La foi dans le Bon (?) Dieu est ébranlée. Lemontey écrit au sujet des survivants : "On use des plaisirs de la richesse avec une ardeur qui tient de la frénésie... Les femmes perdent toute pudeur. " Libérés d'un conjoint souvent imposé par le père, l'oncle ou le tuteur, les survivants se jettent à corps perdu dans des remariages rapides, conclus selon leur choix personnel ou dictés par des intérêts d'ordre économique : 772 mariages dès 1721 , quatre fois plus d'unions qu'en 1720 ! soit 1544 conjoints sur environ cinq mille adultes survivants! Et 611 mariages encore en 1722, soit 1222 nouveaux conjoints. Deux cents mariages pour le seul mois d'octobre 1721, chiffre record. En deux ans, 2766 Toulonnais se sont remariés, soit un Toulonnais adulte sur deux! L'une des autres conséquences de la peste fut de peupler Toulon de non-Toulonnais : en 1719, $10 \%$ des femmes mariées à Toulon étaient nées en dehors de Toulon, 17 \% des hommes. En 1750, 39 \% des femmes mariées à Toulon sont nées en dehors de Toulon, 53 \% des hom-

12. Arch. du port de Toulon, 9 janv. 1723, 1 A 1150. 
mes. Là sont les vraies conséquences de la peste, démographiques et mentales. La ruine économique est aussi un prétexte à la demande de subsides royaux. Plus les Toulonnais paraîtront pauvres, plus le Roi, pense-t-on, se montrera généreux en matière fiscale. En effet, les deux foires franches de quinze jours (29 septembre et 3 novembre) établies à Toulon restent très fréquentées. En 1725, quatre-vingts caboteurs viennent alimenter la foire de novembre. Il est vrai que face au petit nombre d'acheteurs, vingt seulement reviennent en 1726 .

Du côté de la marine de guerre, la ruine est réelle : les armements deviennent peu fréquents et peu importants en nombre, trois ou quatre vaisseaux; les campagnes sont dirigées contre les Barbaresques et tournent parfois au tragique avec prise en otage des officiers français, débarqués en Afrique du Nord tel M. de Mons! Toulon arme quelques unités à destination du Maroc (1720), de Tunis, d'Alger et de Tripoli bombardée en 1728 par M. de Grandpré. Les expéditions glorieuses font défaut. L'avancement piétine : Joseph de Thomas, " marquis " de Châteauneuf (Toulon 1675-Toulon 1753), reste vingt ans enseigne de vaisseau (1703) avant d'être promu lieutenant (1723), et comme beaucoup de camarades, il préfère se retirer (1728), à cinquante-trois ans, avec une petite pension annuelle, cinq cents livres sur les fonds de la Marine et trois cents sur la caisse des invalides ${ }^{13}$. Lieutenant des maréchaux de France à Toulon (1730), le "marquis " sans marquisat mourut à Toulon dans la nuit du 31 janvier 1753. M. de Villeblanche, intendant du port, informa la Cour, le lendemain, de la mort de cet ancien lieutenant de vaisseau de soixante-dix-huit ans.

Carrières ratées. Fortunes médiocres. Pensions faibles. L'heure est à la morosité. Les officiers privés d'avancement et d'embarquements se retirent. Les infrastructures du port se dégradent. Les vaisseaux pourrissent, échoués sur la rive de Ponche-Rimade. Plusieurs sont démolis. En 1719, seuls quatre vaisseaux et deux frégates restent entretenus. Néanmoins les responsables veillent : en 1724, "les deux fils Coulomb sont toujours sur les ouvriers et les savent conduire aussi bien que leur père ${ }^{14}$ ". En 1728, les calfats de Toulon apparaissent au commissaire général d'Héricourt comme supérieurs à tous les calfats " qu'il avait vu pratiquer dans aucun port ". Mais l'époque n'est ni aux réparations, ni à la construction. De 1725 à 1727, le Roi fait détruire à Toulon onze bâtiments : le Sceptre, le Trident et le Fleuron, le Vainqueur, l'Intrépide et le Triomphant, le Bizarre, le Diamant, le Furieux, le Téméraire et le Vermandois. Le bois manque : "La construction du Phoenix est toujours interrompue faute de bois ${ }^{15}$. " L'intendant écrit au Conseil de Marine : "Je ne puis croire que le Conseil s'accommode de la manière de travailler du sieur Levasseur qui emploie un tiers au moins plus de bois qu'un autre dans un temps où ils sont si rares ${ }^{16}$. " En 1722-1723, le

13. Arch. nat., C 7321.

14. Arch. du port de Toulon, 10 sept. 1724, 1 A 1151.

15. Arch. du port de Toulon, août 1722, 1 A 1146.

16. Arch. du port de Toulon, 17 mai 1722, 1 A 1146. 
port réussit à lancer le Solide et le Duc d'Orléans. De 1726 à 1740, quatre vaisseaux sont mis en chantier, offrant du travail aux ouvriers dont le nombre a diminué de $50 \%$ depuis 1721. En 1728, l'intendant Mithon " essaie de vaincre le préjugé contre les bois d'Italie et traite avec le sieur Grancelli de Gênes un achat de trente-six mille pieds cubes de bois par an ". Se procurer du bois devient l'une des priorités de Maurepas, d'où l'intérêt de la France pour "le royaume de Corse " dont Gênes est suzeraine. En 1738, François Chapelle s'y rend ${ }^{17}$. D'où aussi le grand souci de protection des forêts françaises : le 27 janvier 1731, le parlement de Provence autorise seulement cent trente-trois localités à avoir des chèvres car elles abîment trop les forêts. S'intéressent à la question des bois le chef des constructions Levasseur en 1724 et Steirrein maître charpentier ${ }^{18}$, ou en 1736 le constructeur entretenu Jacques-Luc Coulomb qui travaille dans l'arsenal mais construit aussi des bâtiments de commerce ${ }^{19}$, et plus tard, en 1760, Joseph-Marie-Blaise Coulomb, constructeur entretenu.

Ces années difficiles sont marquées par le triste spectacle qu'offre la municipalité. Élu premier consul, M. de Ricard-Tourtour, officier de marine infirme, décline le poste. Le second consul Flamenq (d'une famille anoblie en 1707 par Louis XIV lors du siège du port par les Impériaux), brigue aussitôt la place. Mais un nouveau premier consul est élu, M. de Gérin, officier de marine, qui décline l'offre. L'ambitieux Flamenq ayant rossé de coups le troisième consul Granet pour lui arracher les clefs du coffre de la communauté, le Roi nomma Jean d'Antrechaux, premier consul à nouveau. Mais le nouveau conseil ne put entrer en fonction qu'après six mois de flottement (15 juin 1728-1 ${ }^{\text {er }}$ janvier 1729). Le tout dans un contexte difficile; le pain manque : il faut acheter mille charges de blé de Bourgogne; l'artisanat stagne : d'Antrechaux propose d'ouvrir des fabriques de bas de soie. Quelques fêtes égayent un peu la morosité ambiante. Le 15 septembre 1729, trois fontaines de vin coulent tout un jour pour célébrer la naissance de Monseigneur le Dauphin. Le peuple dîne, repas de midi, dans les rues. Le soir, les consuls font allumer des feux de joie. Le 16, des joutes sont dotées de prix : dix pistoles, une épée, un chapeau bordé, une écharpe; un dîner de soixante couverts est offert par les consuls à M. Dupont, commandant de la place; il fallut ensuite nettoyer les rues pendant quatorze jours! Le 12 janvier 1729, profitant de son entrée en fonction, d'Antrechaux se remarie et épouse Anne-Paule de Grimaudet de Motheux ${ }^{20}$, fille de Louis, capitaine de vaisseau et de feue Claire Daniel de Lhéry de La Seyne, mariage célébré en la chapelle Saint-Jean de l'hôtel de ville. Les collègues du consul furent ses témoins. Union bénie des Dieux : le 18 janvier 1730, Louis-Victor est le premier des huit enfants du couple. D'Antrechaux, sorti de charge le $1^{\mathrm{er}}$ janvier 1730, rentre alors dans la vie domestique pour dix ans. À la même époque

17. Arch. du port de Toulon, 22 juin 1738,1 A 1178.

18. Arch. du port de Toulon, 7 mai 1724, 1 A 1151.

19. Arch. nat., C7 74.

20. Morte veuve en 1780 . 
(1725), le capitaine de vaisseau Antoine Clavel, fils d'officier de vaisseau, épouse Esther de Terras ${ }^{21}$ et en 1730 meurt à Toulon le vieux capitaine de vaisseau Louis de La Boissière de Mornay (v.1655-1730), 85 ans, fils de Ninon de Lenclos et du marquis de Villarceaux.

Le Toulon de Maurepas végète, réduit à ces noces villageoises et à ces quelques cérémonies funèbres. Du côté de la ville, les travaux sont rares : une ayguade est aménagée devant l'hôtel de ville à partir de 1730, pour permettre aux bâtiments de la marine de s'y avitailler. Du côté de l'arsenal, Toulon en 1730 n'a qu'une dizaine de vaisseaux et cinq frégates en état. En 1731, la marine achète au Commerce le Comte de Morville, construit en 1724 et rebaptisé l'Heureux, pour à peine plus de 80000 livres. Seule l'arrivée de Duguay-Trouin (1673-1736), corsaire malouin nommé commandant de la Marine à Toulon, donne au port un peu de relief. Duguay-Trouin prépare à Toulon l'escadre qu'il conduit à Alger, quoique souffrant. Le célèbre savant La Condamine est à son bord. Duguay-Trouin stimule les officiers toulonnais et apprécie particulièrement le capitaine de port Louis Beaussier; mais ce dernier meurt à Toulon le 11 avril 1731. L'illustre Breton protège alors ses fils, petits-fils du feu maître d'équipage Vincent Beaussier.

Dans toutes ces familles "montantes " toulonnaises, la Marine s'est imposée depuis Colbert comme un débouché héréditaire. Les années 1730 voient monter aux grades la troisième génération de ces familles. Ainsi Joseph de Thomas, "marquis " de La Valette (1672-1744), qui se contente d'être présent à Toulon à chaque revue, il y touche régulièrement ses cent cinquante livres mensuelles, fait-il admettre son fils unique dans la Marine : garde-marine en 1723, il est promu enseigne de vaisseau en 1727. Et le " marquis " l'embarque avec lui sur le Ferme ${ }^{22}$ pour une campagne à Tunis et Alger, puis sur l'Alcion qu'il commande ${ }^{23}$, avec pour commandant en second Pierre de Thomas, futur commandeur de Châteauneuf (1684-1759), dans l'escadre de Duguay-Trouin pour une campagne en Barbarie (Alger, Tunis, Tripoli). Tous ces officiers, Beaussier, Thomas, Signier de Piosin, Grimaudet de Motheux, participent aux mêmes campagnes. Le " marquis " de La Valette commande (1734) le Léopard ${ }^{24}$, dans l'escadre de Court de La Bruyère (campagne à Alger, Gibraltar, Cadix), puis à nouveau ${ }^{25}$ dans l'escadre de Beaucaire passée (1735) aux ordres du marquis d'Antin, petit-fils de $\mathrm{M}^{\text {me }}$ de Montespan, après l'attaque d'apoplexie de celui-ci (campagne à Cadix). La Valette commande ensuite le Conquérant ${ }^{26}$, dans l'escadre de La Roche-Allard et subit une forte épidémie de fièvre jaune aux Antilles alors que s'éveille la future guerre de Succession d'Autriche.

21. Mariage de 1725 (Sainte-Marie). Morte à Toulon à vingt-neuf ans (1727, Sainte-Marie). Son mari vit en 1755, chevalier de Saint-Louis. Arch. com. Toulon, registres paroissiaux 1725, 1727, 1755.

22. 9 mai $1727-17$ sept. 1727.

23. 25 mai $1731-1^{\text {er }}$ nov. 1731 .

24. 14 mai $1734-23$ oct. 1734 .

25. 3 août 1735-26 janv. 1736 .

26. 20 août $1740-20$ avril 1741 . 
Dans les années qui précèdent celle-ci, Toulon renoue avec une certaine activité. En 1734, Toulon arme pour la Baltique, lorsqu'il s'agit pour Louis XV de tenter de rétablir son beau-père Stanislas sur le trône de Pologne. Mais la guerre interrompt les coupes de bois dans la péninsule Italienne parce que le bois doit passer par des places appartenant à l'Empereur. Toulon, qui commandait en 1702, 35000 pieds cubes dans la péninsule Italienne et en Catalogne, et qui commandait huit millions de pieds cubes en Italie livrables par lot annuel de 500000 , est contraint de se rabattre sur les frontières de l'Est : en 1735, Toulon commande 50000 pieds cubes de bois de Lorraine, en 1736, 150000 pieds. Ce n'est qu'à la fin de la décennie 1730 que le port connaît un réel frémissement, aussi bien en ville que dans l'arsenal. Mais il fallut attendre quarante années encore pour que la population toulonnaise " récupère " ses effectifs de 1720 décimés par la peste, d'où peut-être les malheurs de la guerre de Sept Ans (1756-1763), qui ne seront oubliés que lorsque le bailli de Suffren, rentré de l'Inde en héros, viendra à Toulon manger la reproduction en sucre de son vaisseau-amiral, au milieu des acclamations et vivats populaires, sur des quais couverts de fleurs et d'arcs de triomphe. Là les malheurs de la peste de 1720-1721 seront alors vraiment oubliés, cinq ans avant que la Révolution ne vienne à nouveau bousculer le cours habituel du Temps. 


\section{RÉSUMÉ}

Toulon connaît les épidémies depuis toujours : 1348, 1581, 1587, 1664. D'où la création de Bureaux de Santé à Marseille et Toulon, ce dernier dès 1576. Celui-ci instaura à Lagoubran - à l'ouest de Toulon -, un lazaret qui devait permettre de faire accoster les bateaux dans un abri jusqu'à la fin de leur mise " en quarantaine "; néanmoins ce lazaret fut plus un mouroir qu'un établissement de soin. La peste de 1720-1721 le prouva. Début octobre 1720, les premiers cas de peste apparaissent à Toulon. Mi-octobre, les premiers morts. En février 1721, les morts remplissent un tombereau quotidien : cinquante places. En mars, deux tombereaux deviennent nécessaires pour évacuer les cadavres : cent morts par jour. La Marine organise son périmètre de sécurité et prend une décision originale puisqu'elle décide d'enfermer les " jeunes ", c'est-à-dire les gardes de la marine au " Jardin du Roi ». Tous survivront à l'exception d'un Moscovite, envoyé à Toulon par Pierre le Grand, et imprudemment sorti du " Jardin du Roi ". En avril, quatre tombereaux quotidiens s'avèrent nécessaires : deux cent soixante et dix morts sont à déplorer pour le 30 avril. Début mai, huit tombereaux quotidiens deviennent indispensables : quatre cents morts quotidiens. L'épidémie culmine. En vain, on cherche des remèdes. On pend quelque galérien et quelques filles de joie pour tenter d'exorciser le mal. Ici, un capucin multiplie les prêches. Là, l'évêque de Toulon voue Toulon au Sacré-Coeur. Le 10 mai, la " serrade " est levée. Le 30 octobre, un Te Deum réunit les survivants : environ 10000 habitants sur 25000 . La peste fut une vraie catastrophe; démographique, culturelle, quantités de " savoirs " ayant été emportés. Toulon est au plus bas : le commerce ruiné, l'artisanat en déroute. La ruine est autant morale qu'économique. Il fallut attendre quarante années pour que la population toulonnaise " récupère " ses effectifs de 1720 décimés par la peste.

\section{ABSTRACT}

Toulon has always experienced epidemics. Such was the case in 1348, 1581, 1587 and 1664 hence the setting up of boards of health in Marseilles and Toulon. The latter boasted one as early as 1576. A lazaretto was established in Lagoubran, west of Toulon. It was supposed to have allowed ships to berth in a shelter until their quarantine had come to an end but that lazaretto was more of a place where people were left to die than a medical care institution. The plague of 1720-1721 gave proof of it. In early October 1720, the first cases of plague appeared in Toulon. By mid October, there were the first dead. In February 1721 the dead filled a fifty-body tip cart on a daily basis. In March, two tip carts became necessary to get rid of corpses : one hundred people died every day. The Navy planned its safety zone and made an original decision since it decided to confine the "young ones", that is the navy guards to "the King's Garden". All of them were to survive except for one Muscovite who had been sent to Toulon by Peter the Great and had recklessly left the "King's Garden". In April, four daily tip carts proved to be necessary: sadly, there were two hundred and seventy dead by April $30^{\text {th }}$. In early May, eight tip carts became indispensable: there were four hundred dead each day. The epidemic then reached its peak. Remedies were looked for but to no avail. A few galley slaves and a few ladies of the night were hanged to try and exorcize evil. Here, a Capuchin friar read repeated sermons. There, the Bishop of Toulon vowed Toulon to the Sacred Heart. On May 10th, the quarantine ("serrade") was lifted. On October $30^{\text {th }}$, a Te Deum gathered the survivors: roughly 10000 inhabitants out of 25000. The plague was a real catastrophe both demographically and culturally speaking. A great deal of "know-how" had been taken away. Toulon was at a very low ebb: its trade was ruined; its crafts had collapsed. The ruin was both moral and economic. Forty years elapsed before the population of Toulon was able to recover the numbers of 1720 which had been decimated by the plague. 